Brief paper

\title{
A numerical method for stability windows and unstable root-locus calculation for linear fractional time-delay systems
}

\author{
André Ricardo Fioravanti ${ }^{\mathrm{a}, 1}$, Catherine Bonnet ${ }^{\mathrm{a}}$, Hitay Özbay ${ }^{\mathrm{b}}$, Silviu-Iulian Niculescu ${ }^{\mathrm{c}}$ \\ ${ }^{a}$ INRIA Saclay - Ilê-de-France, Supélec, 3 rue Joliot Curie, 91192, Gif-sur-Yvette, France \\ ${ }^{\mathrm{b}}$ Bilkent University, Department of Electrical and Electronics Engineering, Ankara 06800, Turkey \\ ${ }^{\mathrm{c}}$ L2S (UMR CNRS 8506), CNRS-Supélec, 3 rue Joliot Curie, 91192, Gif-sur-Yvette, France
}

\section{A R T I C L E I N F O}

\section{Article history:}

Received 3 March 2011

Received in revised form

28 October 2011

Accepted 18 November 2011

Available online 14 August 2012

\section{Keywords:}

Delay effects

Fractional systems

Neutral systems

Root-locus

\begin{abstract}
A B S T R A C T
This paper aims to provide a numerical algorithm able to locate all unstable poles, and therefore the characterization of the stability as a function of the delay, for a class of linear fractional-order neutral systems with multiple commensurate delays. We start by giving the asymptotic position of the chains of poles and the conditions for their stability for a small delay. When these conditions are met, the root continuity argument and some simple substitutions allow us to determine the locations where some roots cross the imaginary axis, providing therefore the complete characterization of the stability windows. The same method can be extended to provide the position of all unstable poles as a function of the delay.
\end{abstract}

(c) 2012 Elsevier Ltd. All rights reserved.

\section{Introduction}

The presence of delays in a system, usually, is a source of poor performance and instability. Therefore, they have been an important subject both in the theoretical and practical domains, as indicated by the large amount of articles dealing with many different problems for this class of systems. Starting from the studies of Pontryagin (1955), and Bellman and Cooke (1963), many results have been achieved, specially over the past decades, as it is discussed, among many others, in the books $(\mathrm{Gu}$, Kharitonov, \& Chen, 2003; Niculescu, 2001; Niculescu \& Gu, 2004) and the survey paper (Richard, 2003), and references therein. Analogously, fractional order systems are also obtaining large attention in the literature of the past years, mainly because they offer an excellent fit to the data in many practical situations as, for example, in biophysics, thermodynamics and rheology; see Hilfer (2000) and references therein.

\footnotetext{
The material in this paper was presented at the 18th IFAC World Congress, August 28-September 2, 2011, Milano, Italy. This paper was recommended for publication in revised form by Associate Editor Andrea Serrani under the direction of Editor Miroslav Krstic.

E-mail addresses: fioravan@dsce.fee.unicamp.br (A.R. Fioravanti), catherine.bonnet@inria.fr (C. Bonnet), hitay@bilkent.edu.tr (H. Özbay), Silviu.Niculescu@lss.supelec.fr (S.-I. Niculescu).

1 Tel.: +55 019 35213864; fax: +5501935213847.
}

When dealing with any dynamical systems, one of the basic questions we need to answer is its stability. For the systems with delay, we can go even further, and be interested on how this property will vary if we increase the value of the delay. When dealing with the non-fractional case, it is known that an interesting phenomenon, namely stability windows, might happen (Walton \& Marshall, 1987). This implies that, even if the delay-free system is unstable, we might find some regions in the delay space where the system is stable. Later, we will see that the same fact can happen for fractional systems. There has been a large effort to deal with this problem for the standard case; see Walton and Marshall (1987), and Olgac and Sipahi (2004) and others.

The most natural way to find the position of the poles of a linear system is to solve its corresponding characteristic equation. But in our case this will be a transcendental one, being thus generally impossible to solve it directly. For this reason, most of the existing procedures study stability of such systems by finding the crossings of poles through the imaginary axis. This fact comes from two important properties of time-delay systems, also valid for the class of fractional systems. The first one is the root continuity argument, which means that for any positive value of the delay, the position of the poles varies continuously with respect to delay. This means that any root crossing from the left half plane to the right half-plane will need to pass through the imaginary axis. The second property is the invariance of the tendency of roots crossing (Olgac \& Sipahi, 2002). This implies that a manageable number of root clusters can 
provide sufficient information to characterize the whole stability of the system.

The fractional case is much more involved, and normally cannot be solved by the methods involving the Routh-Hurwitz table, as the one presented in Olgac and Sipahi (2002). To the best of the authors' knowledge, only the method of Walton and Marshall (1987) can be successfully expanded to cope with fractional systems with multiple delays, see Bonnet and Partington (2002), but each extra commensurate term of the delay after the first one needs to be reduced, and this process potentially doubles at each step the degree of the polynomial we need to solve. This implies that even if we are dealing with a low degree system with multiple delays, this method will require the zeros of a polynomial with high order, which can be a challenging and unreliable numerical problem.

Recently, there has been developments of new methods dealing with the stability of the fractional order system with delays. In Hwang and Cheng (2006), a numerical procedure based on Cauchy's integral theorem was proposed to test the stability of such systems, and in Hwang and Cheng (2005), a technique based on the Lambert $W$ function was used for the same purpose. But the complete characterization of all stability windows is difficult when using those methods, and no information about the position of the unstable poles are given.

When dealing with neutral systems, one needs to take extra care due to the small-delay effect, as it is presented in Hale and Verduyn Lunel (2001) for the non-fractional case. This phenomenon is also present for fractional delay systems (Bonnet, Fioravanti, \& Partington, 2011; Bonnet \& Partington, 2002) for the single and multiple delay cases, respectively. This effect splits the set of delay systems into two classes. The first one presents the same number of unstable roots when the delay passes from 0 to $0^{+}$, and therefore has a chance of being stable for a non-zero value of the delay, while, in the second one, infinitely many unstable roots appear when the delay varies from 0 to $0^{+}$. Even if the delay free system is stable, systems of the second class cannot recover stability for any finite positive value of the delay.

The rest of the paper is organized as follows. Section 2 contains the problem statement and assumptions. Section 3 brings some stability tests for the type of systems we are dealing with, and in Section 4, based on a pseudo-delay transformation, we develop a numerical procedure that identifies the stability zones as a function of the delay. The location of the unstable roots as well as their variation as a function of the delay is also given. Section 5 brings some examples to illustrate the results presented and finally Section 6 concludes the work.

The notation used throughout is standard. The set of natural numbers is denoted by $\mathbb{N}$, whereas $\mathbb{N}_{N}$ denotes the set of its first $N$ elements (i.e., $\left.\mathbb{N}_{N}=\{1, \ldots, N\}\right)$. $\mathbb{C}\left(\mathbb{C}^{+}, \mathbb{C}^{-}\right)$is the set of complex numbers (with strictly positive and strictly negative real parts), and $\jmath=\sqrt{-1}$ is the imaginary unit. For $z \in \mathbb{C}, \bar{z}$ denotes its complex conjugate, and $\angle z, \Re(z)$ and $\mathfrak{I}(z)$ define the argument (taken here from $(-\pi, \pi])$, the real part and the imaginary part of $z . \mathbb{R}\left(\mathbb{R}^{+}, \mathbb{R}^{-}\right)$denotes the set of real numbers (larger or equal to zero, smaller or equal to zero).

\section{Problem formulation}

We will consider a system that has in the frequency-domain the following characteristic equation:

$C(s, \tau)=p\left(s^{\alpha}\right)+\sum_{k=1}^{N} q_{k}\left(s^{\alpha}\right) e^{-k s \tau}$,

where the parameter $\tau$ is non-negative, $p\left(s^{\alpha}\right)$ and $q_{k}\left(s^{\alpha}\right)$ for $k \in \mathbb{N}_{N}$ are polynomials in $s^{\alpha}$ with $\alpha \in(0,1)$ and $\operatorname{deg} p \geq \operatorname{deg} q_{k}$. Here, the degree is interpreted as the degree in $s^{\alpha}$, and therefore it is an integer. If $\operatorname{deg} p=\operatorname{deg} q_{k}$ for at least one $k \in \mathbb{N}_{N}$, then Eq. (1) defines a neutral time-delay system, otherwise it will consist of retarded type. As it will be seen in sequence, the analysis of the former is more involved and will require one extra step.

Clearly, (1) is the characteristic equation of a feedback system whose open loop transfer function is

$G(s)=\frac{1}{p\left(s^{\alpha}\right)} \sum_{k=1}^{N} q_{k}\left(s^{\alpha}\right) e^{-k s \tau}$.

The poles of the closed loop transfer functions $(1+G)^{-1}$ and $G(1+G)^{-1}$ are the roots of (1). So, in what follows, sometimes refer to the roots of (1) as "poles". Furthermore, we assume the following.

Assumption 1. The polynomials $p(s), q_{1}(s), \ldots, q_{N}(s)$ do not have any common zero.

It is obvious that if Assumption 1 is violated, then a common factor $c(s) \neq \mathrm{a}$ constant can be factored out of the characteristic polynomial (1). These poles will be present for all the values of $\tau$, and therefore, if any of the poles of $c(s)$ is unstable, the system will be unstable for all values of the delay, whereas if all the poles are stable, they do not play a significant role in the stability test. In this case, simplifying by $c(s)$ we get a system described by (1) satisfying Assumption 1.

Assumption 2. The polynomials $p(s)$ and $q_{k}(s)$ satisfy

$p(0)+\sum_{k=1}^{N} q_{k}(0) \neq 0$.

This means that $s=0$ is not a roof of (1) for all values of $\tau$. Simultaneously, this assumption also guarantees that poles at $s=$ 0 can only happen for $\tau \rightarrow \infty$. This property will be very important in the following sections.

\section{Stability of fractional-order systems with delay}

We work here in an input-output framework and consider $H_{\infty}$-stability, that is, the system has a finite $L_{2}(0, \infty)$ gain if

$\|G\|_{H_{\infty}}=\sup _{u \in L^{2}, u \neq 0} \frac{\|G u\|_{L^{2}}}{\|u\|_{L^{2}}}<\infty$,

and we recall that $H_{\infty}\left(\mathbb{C}_{+}\right)$is the space of functions which are analytic and bounded in the open right half-plane $\mathbb{C}_{+}$.

For fractional-order systems without delay, stability was first demonstrated in Matignon (1998). A practical test for stability can be achieved if we use the variable substitution $\varsigma=s^{\alpha}$. Applying this substitution, the characteristic Eq. (1) becomes

$C_{\varsigma}(\varsigma, \tau)=p(\varsigma)+\sum_{k=1}^{N} q_{k}(\varsigma) e^{-k \varsigma^{1 / \alpha} \tau}$,

and therefore, when $\tau=0$, it results in

$C_{\varsigma}(\varsigma, 0)=p(\varsigma)+\sum_{k=1}^{N} q_{k}(\varsigma)$.

This substitution will transform the domain of the system from a multi-sheeted Riemann surface into the complex plane, where its poles can be easily calculated. In this new variable, the instability region of the original system is not given by the right half-plane, but in fact by the region described as

$|\angle \varsigma| \leq \alpha \frac{\pi}{2}$

with $\varsigma \in \mathbb{C}$, as illustrated by Fig. 1 . 


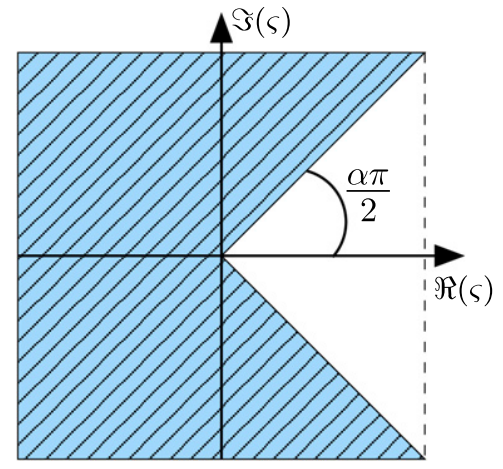

Fig. 1. The $\varsigma$-stability region for fractional systems.

Notice that under this transformation, the imaginary axis in the $s$-domain is mapped into the lines

$\angle \varsigma= \pm \alpha \frac{\pi}{2}$

in the $\varsigma$-domain, and therefore a solution $\varsigma^{\star}=\left|\varsigma^{\star}\right| \angle \pm \alpha \pi / 2$ implies that the original system has a purely imaginary solution of the type

$s^{\star}= \pm j\left|\varsigma^{\star}\right|^{\alpha^{-1}}$.

Also, we can notice that every solution $\varsigma^{*}$ such that $\left|\angle \varsigma^{*}\right|<\alpha \pi$ is mapped into the physical Riemann sheet in the $s$-domain through the inverse transform $s=\varsigma^{1 / \alpha}$.

The BIBO-stability (i.e., the system presents a finite $L_{\infty}$-gain) of fractional systems with delays has been considered in Bonnet and Partington (2002) where it is shown that BIBO stability conditions already known for delay systems can be extended to the case of fractional delay systems. Their results are summarized in the next two lemmas.

Lemma 3. Let $G$ be a strictly proper system with characteristic equation given by (1) satisfying $\operatorname{deg} p>\operatorname{deg} q_{k}$ for $k \in 1, \ldots, N$, being thus of retarded type. Then $G$ is BIBO-stable if and only if $G$ has no poles in $\{\Re(s) \geq 0$ \} (in particular, no poles of fractional order at $s=0)$.

Lemma 4. Let $G$ be a strictly proper system with characteristic equation given by (1) satisfying $\operatorname{deg} p \geq \operatorname{deg} q_{k} k \in 1, \ldots, N$ with the equality holding for at least one polynomial $q_{k}$, being thus of neutral type. If there exists $a<0$ such that $G$ has no poles in $(\mathbb{C} \backslash \mathbb{R}) \cap\{\Re(s)>$ a\} $\cup\{0\}$ then $G$ is BIBO-stable.

As it is known that BIBO-stability implies $H_{\infty}$-stability (see Mäkilä and Partington (1993) and Partington and Mäkilä (1994)), similar results can be derived immediately for $H_{\infty}$-stability. Finally, $H_{\infty}$-stability conditions for systems with poles asymptotically approaching the imaginary axis are given in Bonnet et al. (2011), and Partington and Bonnet (2004), among others.

\section{Numerical procedure}

Our first objective of this section is to derive a procedure to calculate, for systems described as in (1), the values of $\tau$ where there exists a crossing of roots through the imaginary axis. We want to stress that under the variable substitution $\varsigma=s^{1 / \alpha}$, this problem is equivalent to find all the values of $\tau$ where there exists a crossing of poles through $\angle \varsigma= \pm \alpha \frac{\pi}{2}$ in $C_{\zeta}(\varsigma, \tau)$.

With these values in hand, we will be able to calculate the direction of crossing from the left half plane to the right one, which we will denote as a destabilizing crossing, or from the right to the left, denoted as a stabilizing crossing. Notice that the use of the expressions destabilizing and stabilizing crossings means only that a pair of poles is crossing the imaginary axis in the defined direction, and not that the system is turning unstable or stable, respectively. For that, it is necessary to know the number of unstable poles before those crossings occur.

\subsection{Stability for $\tau=0$}

The first step for all methods based on the root continuity argument is to start by the study of the delay free system. Considering $\tau=0$ as in (6), we get a polynomial with real coefficients, whose roots can be easily found. We will denote the number of unstable poles of $C_{\zeta}(\varsigma, 0)$ as $N_{u}$ and their location $\varsigma_{k}$ for $k \in \mathbb{N}_{N_{u}}$, since this information will be crucial in the following developments. As it is illustrated in Fig. 1, for this case, an unstable pole is any solution of $C_{\varsigma}(\varsigma, 0)=0$ with $|\angle \varsigma| \leq \alpha \frac{\pi}{2}$, where we recall that $\angle \varsigma \in(-\pi, \pi]$.

\subsection{Location of chains of poles}

If the system we are dealing with is of the retarded type, all the infinite new poles that appear when we pass from $\tau=0$ to $\tau=0^{+}$ will be in the extreme left half-plane, that means, $\Re(s) \rightarrow-\infty$, and so this step can be ignored. The case of neutral systems needs to be dealt with care. We will follow the steps from Bonnet et al. (2011) for the characterization of the asymptotic behavior of the chains of poles.

Proposition 5. Let $C(s, \tau)$ be the characteristic equation of a neutral system defined as in (1). Consider the first order approximation

$$
\frac{q_{k}(s)}{p(s)}=a_{k}+\mathcal{O}\left(s^{-\alpha}\right) \quad \text { as }|s| \rightarrow \infty,
$$

where, denoting $\operatorname{deg} p=d, p(s)=\sum_{m=0}^{d} \rho_{m} s^{m}$ and $q_{k}(s)=\sum_{m=0}^{d}$ $\sigma_{k, m} s^{m}$, Eq. (10) is satisfied with

$a_{k}=\frac{\sigma_{k, d}}{\rho_{d}}$.

Now let $M \leq N$ be the greatest integer such that $a_{M} \neq 0$. The neutral chains of poles asymptotically approach the vertical lines

$\Re(s)=-\frac{\ln (|r|)}{\tau}$

for each solution $r$ of the polynomial equation

$1+\sum_{k=1}^{M} a_{k} z^{k}=0$

Proof. See Bonnet et al. (2011) and references therein.

Eqs. (12) and (13) provide an easy-to-check condition in order for the neutral chains of poles be in the left half-plane. For this, all roots $r$ of (13) must have $|r|>1$.

In case any $|r|<1$, then at least one chain of poles will always be in the right half-plane. That means that the system cannot be stable for any positive value of $\tau$. The intermediate case, where we have all $|r| \geq 1$, with the equality holding for at least one root of (12), needs to be further explored to determine from which side of the imaginary axis the poles are. But it is important to recall that in this case, the rule "no poles in the close right half-plane" is no longer sufficient to guarantee $H_{\infty}$-stability, and other properties must be checked. Indeed, the characteristic equation does not provide the full information necessary to state stability in this case, and we need to work with the complete transfer function. We invite the reader to see Bonnet et al. (2011) for further insights on this subject. 
Assumption 6. From now on, we will consider that there are no chains of poles in the extended right half-plane (i.e., there exists a finite number of poles in $\Re(s)>-a$ for some $a>0$ ), which corresponds to all the solutions of (13) having modulus strictly greater than one.

\subsection{Crossing position}

In order to find the location in the imaginary axis where the crossings occur, we will rely on a transformation of variables which decouples the polynomials and the exponential part. Since for $s=$ $\jmath \omega$ the exponential terms in (1) will have modulus equal to one, the idea is to replace $e^{-j \omega \tau k}$ with $e^{-\jmath \theta k}$ and find the roots of the resulting complex pseudo-polynomial as a function of $\theta \in[0, \pi]$. In other words, we find all the roots of the complex pseudo-polynomial in $s$

$\widetilde{C}(s, \theta)=p\left(s^{\alpha}\right)+\sum_{k=1}^{N} q_{k}\left(s^{\alpha}\right) e^{-j k \theta}$,

by varying $\theta$ in the interval $[0, \pi]$.

Lemma 7. For any $\tau$ and $s=\jmath \omega$ such that $\widetilde{C}(s, \theta)=0$, we have $\widetilde{C}(\bar{s}, 2 \pi-\theta)=0$.

Proof. It is a matter of simple substitution to show that $\widetilde{C}(\jmath \omega, \theta)=$ $\widetilde{\widetilde{C}}(-\jmath \omega, 2 \pi-\theta)$.

Lemma 8. There exist $s=\jmath \omega$ and $\tau>0$ such that $C(s, \tau)=0$ if and only if there exists $\theta \in[0,2 \pi]$ such that $\widetilde{C}(s, \theta)=0$.

Proof. For the sufficiency, recall that by Assumption 2 the case $\omega=0$ can be neglected. So letting

$\tau(\omega, \theta, \ell)=\frac{\theta}{\omega}+\frac{2 \pi \ell}{\omega}$

and choosing $\ell=\{0,1, \ldots\}$ if $\omega>0$ or $\ell=\{-1,-2, \ldots\}$ if $\omega<0$ will provide $\tau>0$ such that (1) is satisfied.

For the necessity, choose $\theta$ as $\angle e^{j \omega \tau}$, taken from $[0,2 \pi]$, and notice that

$e^{-\jmath k \omega \tau}=e^{-\jmath k \theta}$

for all $k \in \mathbb{N}$.

It is important to notice that for a fixed $\theta \in[0, \pi], \widetilde{C}$ is a pseudopolynomial with complex coefficients, but no delays. But as it was stated before, with the variable transformation $s=s^{\alpha}$, there is a direct relation between the roots on the imaginary axis for the $s$-domain with the ones having argument $\pm \alpha \pi / 2$ in the $\varsigma$-domain. We must also recall that, due to the variable transformation in the exponential term, a solution $s^{\star}$ of $\widetilde{C}$ does not imply that $\overline{s^{\star}}$ is another solution. On the other hand, since by Assumption 2 we can neglect the roots at the origin, all roots in the imaginary axis of (1) occur in complex conjugate pairs, and so we can finally state our main result.

Theorem 9. Let Assumptions 1, 2 and 6 hold. Let $\Omega$ be the set of all ordered pairs $(\omega, \theta)$, with $\omega \in \mathbb{R}$ and $\theta \in[0, \pi]$ such that $\widetilde{C}(j \omega, \theta)$ $=0$. Let

$\tau(\omega, \theta, \ell)=\frac{\theta}{\omega}+\frac{2 \pi \ell}{\omega}$

for all $(\omega, \theta) \in \Omega$. Choose $\ell=\{0,1, \ldots\}$ if $\omega>0$, and $\ell=\{-1$, $-2, \ldots\}$ if $\omega<0$. Let $\Delta$ be defined as the set of all the ordered pairs $( \pm \jmath \omega, \tau(\omega, \theta, \ell))$. Then $\Delta$ is the complete set of roots of (1) on the imaginary axis for $\tau>0$.

Proof. First we need to show that any element of $\Delta$ is a root for (1). The result for the term " $+\omega$ " comes directly from the sufficiency of Lemma 8, whereas the " $-\omega$ " comes from the aforementioned fact that the poles in the imaginary axis of (1) occur in complex conjugate pairs. Finally, it lacks to show that any root of (1) is an element of $\Delta$. But from Lemma 7 , we see that for the complex conjugated solutions of $\widetilde{C}(\jmath \omega, \theta)=0$, at least one of the $\theta$ will be in the $[0, \pi]$ interval. The rest follows from the necessity of Lemma 8.

With these results in hand, it is easy to check if a system is stable independent of delay.

Corollary 10. If the system given by (1) is stable for $\tau=0$ and $\Omega=\emptyset$, then the system is stable for all positive values of $\tau$.

Proof. Direct from the fact that there are no roots crossing the imaginary axis.

The set $\Omega$ together with the root tendency of each ordered pair is what we call the root cluster. The root tendency will be better explained in the sequel, but as in the non-fractional case (Olgac \& Sipahi, 2004), it is constant with respect to any sequential crossings in (17).

To be able to use the results of Theorem 9 , we need to be able to find all $\omega \in \mathbb{R}$ and $\theta \in[0, \pi]$ such that $\widetilde{C}(\jmath \omega, \theta)=0$. To this matter, we propose two distinct approaches.

The most direct one consists in sampling $\theta$ in its interval $[0, \pi]$, and for each fixed value $\theta^{\star}$, make the variable substitution $\varsigma=s^{\alpha}$, calculate the roots of the resulting complex polynomial in $\zeta$

$\widetilde{C}_{\zeta}\left(\varsigma, \theta^{\star}\right)=p(\varsigma)+\sum_{k=1}^{N} q_{k}(\varsigma) e^{-j k \theta^{\star}}$.

Although we need to deal with a large number of polynomial equations to solve this problem, two points are relevant. First, all polynomials we need to solve will be of degree $n=\operatorname{deg} p$ (for the system description given in (5)). Second, the root continuity argument still holds for $\widetilde{C}_{\zeta}(\varsigma, \theta)$ as a function of $\theta$. As we have guaranteed that no poles in the origin will happen, this means that plotting the absolute value of the argument of those roots as a function of $\theta$ brings a useful graphic information. Also, as by assumption there are no chains of poles asymptotic to the imaginary axis, these plots will be continuous. Therefore, searching the positions where these curves cross the line $\theta=\alpha \pi / 2$ provide the information to calculate where the actual crossings through the imaginary axis are in the $s$-domain. This allows a better use of Newton's method in order to improve numerical accuracy without increasing the possibility of getting false results.

The second method we propose tries to keep the advantages of the previous one without increasing the computational burden. If $(\varsigma, \theta)$ is a simple root of (18), then a small perturbation on $\theta^{\star}=\theta+\epsilon$ will provide a solution of $\widetilde{C}\left(\varsigma^{\star}, \theta^{\star}\right)=0$ with the form

$\varsigma^{\star}=\varsigma+\sum_{k=1}^{\infty} \lambda_{k} \epsilon^{k}$

where

$\lambda_{1}=\jmath \frac{\sum_{k=1}^{N} k q_{k}(\varsigma) e^{-\jmath \theta k}}{p^{\prime}(\varsigma)+\sum_{k=1}^{N} q_{k}^{\prime}(\varsigma) e^{-\jmath \theta k}}=T(\varsigma, \theta)$.

Here, $p^{\prime}(\varsigma)$ and $q_{k}^{\prime}(\varsigma)$ denote the derivative of the polynomials $p(\varsigma)$ and $q_{k}(\varsigma)$ in $\varsigma$ respectively.

So, a method to provide the same curves as the one calculated with the gridding method is to numerically integrate $T(\varsigma, \theta)$ for $\theta$ varying from 0 to $\pi$ and the starting positions of $\varsigma$ being the roots of $\widetilde{C}_{\varsigma}(\varsigma, 0)=0$, which are the same as the roots of $C_{\zeta}(\varsigma, 0)=0$ already calculated for the test of stability in $\tau=0$. A possible strategy to integrate this function is to predict the next step by means of $T(\varsigma, \theta)$ to be close enough of the real solution and therefore being able to correct it with Newton's method. The size 
of the step can be tuned during execution by regarding the distance of the prediction part and the real solution of the correction one.

The cases where $T(\varsigma, \theta)=0$ or where we have multiple poles can still be dealt with; see Chen, Fu, Niculescu, and Guan (2010a, 2010b). But this will need a higher order analysis or a different approach for the definition of (19). On the other hand, in both cases, when this event happens, we can always stop the numerical integration, deal locally with the gridding method provided before, and re-start the integration method once we achieve simple roots and $T(\varsigma, \theta) \neq 0$.

\subsection{Direction of crossing}

The objective now is to find for each crossing of roots through the imaginary axis, if it is a stabilizing or a destabilizing one. As it was shown in Olgac and Sipahi (2002), this is constant with respect to sequential crossings ( $\ell$ in (17)), and therefore it is denoted as root tendency.

It is more convenient to calculate this point in the $s$-domain, but we can still deal with this problem in the same way we dealt before. Assume that $(s, \tau)$ is a simple root of $C(s, \tau)=0$. For a small variation of $\tau^{\star}=\tau+\epsilon$, a solution of $C\left(s^{\star}, \tau^{\star}\right)=0$ can be found with the form

$s^{\star}=s+\sum_{k=1}^{\infty} \mu_{k} \epsilon^{k}$

where

$$
\begin{aligned}
\mu_{1}=s & \frac{\sum_{k=1}^{N} k q_{k}(s) e^{-\tau s k}}{\alpha s^{\alpha-1} p^{\prime}(s)+\sum_{k=1}^{N}\left(\alpha s^{\alpha-1} q_{k}^{\prime}(s)-\tau k q_{k}(s)\right) e^{-\tau s k}} \\
= & V(s, \tau) .
\end{aligned}
$$

The root tendency is given by $\operatorname{sign}(\Re(V(j \omega, \tau)))$, where $(j \omega, \tau) \in$ $\Delta$. If it is positive, then it is a destabilizing crossing, whereas if it is negative, this means a stabilizing crossing. In case the result is 0 , a higher order analysis is needed, since this might be the case where the root just touches the imaginary axis and returns to its original half-plane.

To confirm the root tendency, we will show that

$\operatorname{sign}\left(\Re\left(V\left(j \omega, \frac{\theta}{\omega}+\frac{2 \pi \ell}{\omega}\right)\right)\right)$

is independent of $\ell$. Notice that for those given values, the exponential term

$e^{-\tau s k}=e^{-\jmath \theta k} e^{-\jmath 2 \pi \ell k}=e^{-\jmath \theta k}$

is independent of $\ell$. So, the only part $\ell$ still appears is in the denominator of (22). But since $\operatorname{sign}(\Re(z))=\operatorname{sign}(\Re(1 / z))$ for $z \in \mathbb{C}-\{0\}$, we can calculate the root tendency over the inverse of $V$, and we readily see that $\ell$ just enters in the imaginary part of it.

From this point, it is easy to determine for each value of the delay if the system has unstable roots or not. Start counting from the number of unstable poles of the delay free system. Sort $\Delta$ by the value of the delay of the crossing, and for each value of it such that the root tendency is positive, add one for the counting of unstable poles (they will always appear in pairs), or subtract one if the root tendency is negative. Repeat the procedure until the maximum of the delay. Finally, identify the values of $\tau$ where the number of unstable poles is zero. Those are the stable regions.

\subsection{Location of unstable poles}

Suppose now that the problem is not finding the values of $\tau$ such that the system is stable, but in fact finding the actual position of the unstable poles for a given value of delay $\tau^{\star}$. This can be achieved by an adaptation of the same techniques used before.
From the definition of $\Omega$, we can calculate a subset $\Delta_{\tau^{\star}}$ of $\Delta$ containing only the elements of $\Delta$ with the delay smaller than $\tau^{\star}$ and with positive root tendency.

To avoid any issues around the Riemann surfaces, it is more adequate to deal with this problem in the $\varsigma$-domain. So assuming that $(\zeta, \tau)$ is a simple root of $C_{\zeta}(\zeta, \tau)=0$, then for a small variation of $\tau^{\star}=\tau+\epsilon$ a solution of $C_{\zeta}\left(\varsigma^{\star}, \tau^{\star}\right)=0$ can be found with the form $\varsigma^{\star}=\varsigma+\sum_{k=1}^{\infty} v_{k} \epsilon^{k}$, where

$$
\begin{aligned}
v_{1} & =\frac{\varsigma^{1 / \alpha} \sum_{k=1}^{N} k q_{k}(\varsigma) e^{-\tau \varsigma^{1 / \alpha} k}}{p^{\prime}(\varsigma)+\sum_{k=1}^{N}\left(q_{k}^{\prime}(\varsigma)-\tau \alpha^{-1} \varsigma^{(1-\alpha) / \alpha} k q_{k}(\varsigma)\right) e^{-\tau \varsigma^{1 / \alpha} k}} \\
& =V_{\varsigma}(\varsigma, \tau) .
\end{aligned}
$$

Integrating $V_{\varsigma}(\varsigma, \tau)$ with respect to $\tau$ for each element of $\left(\left(s^{\circ}\right)^{\alpha}, \tau^{\circ}\right) \in \Delta_{\tau^{\star}}$ for $\tau \in\left[\tau^{\circ}, \tau^{\star}\right]$ and $\left(s^{\circ}\right)^{\alpha}$ as a starting point, together with the unstable poles $\left(\zeta_{k}\right)$ for the delay free system for $\tau \in\left[0, \tau^{\star}\right]$ will generate the curves of the root loci in the $\varsigma$-domain. Applying the inverse transformation $s=\varsigma^{1 / \alpha}$ for all the points in the $\varsigma$ root-loci with argument between $(-\alpha \pi, \alpha \pi)$ generates the root-loci in the physical layer in the $s$-domain. We can even back integrate from the elements of $\Delta_{\tau^{\star}}$ for $\tau \in\left[\tau^{\circ}, \tau^{\bullet}\right]$ in order to see their dynamics before the crossing, although back integrating until $\tau^{\bullet}=0$ can be a bad idea since there is a chance that this might lead to solutions asymptotic to $\mathfrak{R}(s) \rightarrow-\infty$. A good trade-off seems to be choosing $\tau^{\bullet}=\tau^{\circ} / 2$.

This procedure will provide the position of all unstable poles of (1), as well as some information about the stable ones. If one needs more information about the stable ones, one can always perform the same integration procedure over the stable poles for $\tau=0$, the stabilizing crossings, and even also back integrating from a number of future crossings. But no guarantee can be given that those will be the ones closer to the imaginary axis.

\subsection{Complete algorithm}

To recapitulate, the main points of the proposed procedure are summarized here.

Step 1. Calculate the position of the poles for the delay free system.

Step 2. Calculate the roots of (13) in order to get the asymptotic position of the neutral chains of poles.

Step 3 Solve (14) for $\theta \in[0, \pi]$ and find the locations where any of the solutions has angle $\alpha \pi / 2$.

Step 4. With the result of the previous item, calculate the root cluster, the root tendency and the location of all crossings happening before the desired value of delay. This solves the question about the stability of the system.

Step 5. Integrate Eq. (25) from the related points in the $\varsigma$-domain just calculated if they have positive tendency as well as the unstable poles for the delay free system until the target delay. Re-map the final points back to the $s$ domain, which may fall outside the physical Riemann sheet. This will provide the location of all the unstable poles.

\subsection{And what if $\alpha=1$ ?}

All the methodologies presented here can be adapted (in fact, simplified) to deal with the case of non-fractional systems, i.e. $\alpha=$ 1. Initial results of this case were presented in Fioravanti, Bonnet, Özbay, and Niculescu (2010), but the one proposed here is much more involved. One major difference is the fact that roots can cross from one Riemann sheet to the other, which makes the procedure of following the roots in the s-domain much more complicated. 


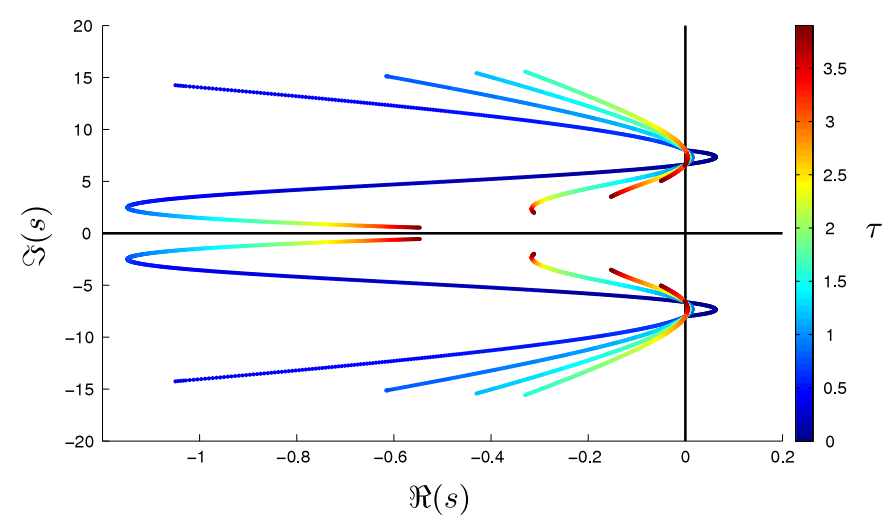

Fig. 2. Root-loci for $C_{1}(s)$ until $\tau=3.9$.

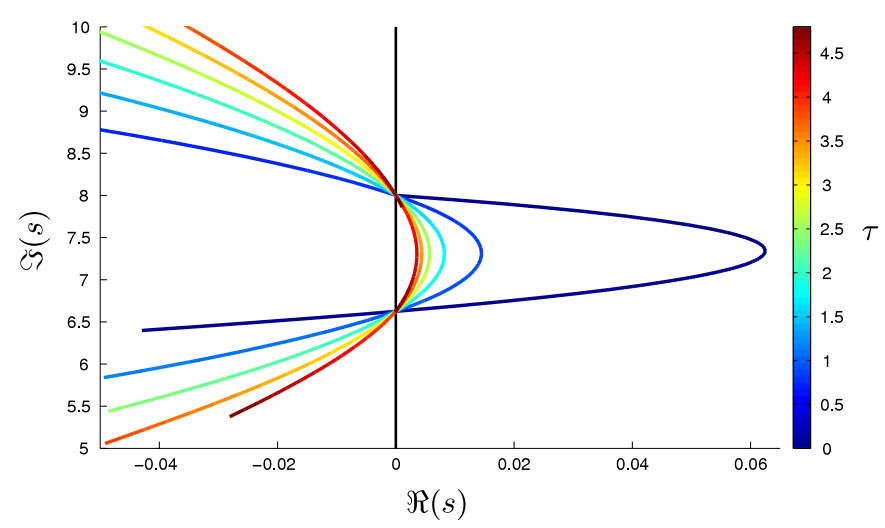

Fig. 3. Zoom at crossing points for $C_{1}(s)$ until $\tau=4.8$.

To circumvent this complexity, we first apply the transformation to work in the $\varsigma$-domain, and after completing the root-locus we map just part of the result which will fall back into the physical Riemann sheet of the $s$-domain. But this transformation changes the form of the delay term, and this must be taken into account.

\section{Examples}

Our first example comes from Hwang and Cheng (2006). Let us consider the system defined by

$$
C_{1}(s)=\frac{1}{(\sqrt{s})^{3}-1.5(\sqrt{s})^{2}+4 \sqrt{s}+8-1.5(\sqrt{s})^{2} e^{-\tau s}} .
$$

Utilizing a heavy computation scheme based on the Cauchy's integral, Hwang and Cheng (2006) showed that this system is unstable for $\tau=0.99$ but stable for $\tau=1$.

Applying the first part of the algorithm, we can see that there is a destabilizing crossing of roots at $\tau=0.7854 \mathrm{k}$ occurring at $s= \pm \jmath 8.0$ and a stabilizing crossing at $\tau=0.0499+0.9485 k$ for $s= \pm \jmath 6.6246$, for all $k \in\{0,1, \ldots\}$. Therefore, we have the following 5 stability windows: $0.0499<\tau<0.7854,0.9983<$ $\tau<1.5708,1.9486<\tau<2.3562,2.8953<\tau<3.1416$ and $3.8437<\tau<3.9270$, which agrees with the result given by Ozturk and Uraz (1985) and in some sense explains it.

We further continue by considering $\tau=3.9$. As this value is inside the last stability window, we know beforehand that the system is stable. But we search a better understanding of the rootlocus of the system as a function of the delay. Fig. 2 brings this picture, where the colors represent the chosen $\tau$, with deep-blue for $\tau=0$ and strong red for $\tau=3.9$.

One can see that although the depth inside the right halfplane for each destabilizing cross becomes less expressive when $\tau$ increases, the stabilizing crossing happens closer to the following destabilizing one, and after the pair of poles cross at $\tau=3.9270$ to the right half-plane, the next pair of unstable poles arrive at $\tau=$ 4.7124 before the previous ones exit this half-plane at $\tau=4.7922$, and therefore the system cannot recover its stability. This can be better seen in Fig. 3, where it is shown a zoom around the crossing points through the imaginary axis.

The second example also comes from Hwang and Cheng (2006). Considering the fractional-order system with two delays

$C_{2}(s)=\frac{1}{s^{5 / 6}+\left(s^{1 / 2}+s^{1 / 3}\right) e^{-0.5 s}+e^{-s}}$.

It is stated that this system is stable. In order to apply our procedure, we transformed $C_{2}(s)$ into the following equivalent system

$\widetilde{C}_{2}(s, \tau)=\frac{1}{s^{5 / 6}+\left(s^{3 / 6}+s^{2 / 6}\right) e^{-\tau s}+e^{-2 \tau s}}$

and we have to study the stability for $\tau=0.5$.

This system has no unstable pole for $\tau=0$ (in fact, it has no pole in the physical Riemann sheet). Applying the methodology described before, we achieve that crossings through the imaginary axis happens for $\tau=2.3562+6.2832 k$ and $\tau=2.6180+6.2832 k$ for all $k \in\{0,1, \ldots\}$, and both of them are destabilizing crosses. This means that the only stability window for this system is $0 \leq$ $\tau<2.3562$. As $\tau=0.5$ is included in this window, we can ensure that the original system $C_{2}(s)$ is stable.

Our last example is adapted from Marshall, Górecki, Korytowski, and Walton (1992). Let $C_{3}(s, \tau)=s^{1.8}+4 s^{0.9}+4-$ $(1 / 4) e^{-s \tau}$. The delay-free system has no poles in the unstable region, and since this is a retarded time-delay system, we do not have issues with the chains of poles. 


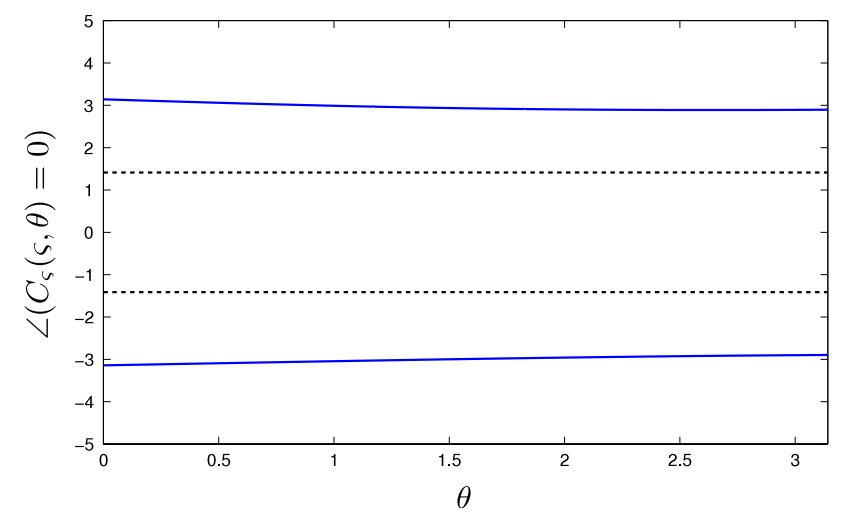

Fig. 4. Angle of the location of poles of $\widetilde{C}_{3}(s, \theta)$.

Fig. 4 shows the angle of the solution of (18) as we vary $\theta$ in the interval $[0, \pi]$. As we can see from the image, the critical lines $\pm 0.45 \pi$ are never crossed, meaning that no poles of the original system will ever pass through the imaginary axis. As the original system was delay-free stable, the time-delay system will be stable for all values of the delay.

\section{Conclusion}

A new method for calculating stability windows and location of the unstable poles is proposed for a large class of fractional order time-delay systems. As the main advantages, we just deal with polynomials of the same order as that of the original system, and we have a graphical representation of the number of elements in the root cluster, which can provide an important visual information for some cases. However, if the delays or the fractional order are not commensurate, the analysis does not apply in its present form. Current work considers how are the best practices to implement the methodology developed, and the results seem promising and consistent with the existing literature.

\section{References}

Bellman, R., \& Cooke, K. L. (1963). Differential-difference equations. New York-London: Academic Press.

Bonnet, C., Fioravanti, A. R., \& Partington, J. R. (2011). Stability of neutral systems with multiple delays and poles asymptotic to the imaginary axis. SIAM Journal on Control and Optimization, 49(2), 498-516.

Bonnet, C., \& Partington, J. R. (2002). Analysis of fractional delay systems of retarded and neutral type. Automatica, 38, 1133-1138.

Chen, J., Fu, P., Niculescu, S.-I., \& Guan, Z. (2010a). An eigenvalue perturbation approach to stability analysis, part I: eigenvalue series of matrix operators. SIAM Journal on Control and Optimization, 48(8), 5564-5582.

Chen, J., Fu, P., Niculescu, S.-I., \& Guan, Z. (2010b). An eigenvalue perturbation approach to stability analysis, part II: when will zeros of time-delay systems cross imaginary axis? SIAM Journal on Control and Optimization, 48(8), 5583-5605.

Fioravanti, A.R., Bonnet, C., Özbay, H., \& Niculescu, S.-I. (2010). A numerical method to find stability windows and unstable poles for linear neutral time-delay systems. In Proceedings of the 9th IFAC workshop on time delay systems.

Gu, K., Kharitonov, V. L., \& Chen, J. (2003). Stability of time-delay systems. Boston: Birkhauser.

Hale, J. K., \& Verduyn Lunel, S. M. (2001). Efects of small delays on stability and control. Operator Theory; Advances and Applications, 122, 275-301.

Hilfer, R. (Ed.) (2000). Applications of fractional calculus in physics. Singapore: World Scientific.

Hwang, C., \& Cheng, Y. C. (2005). A note on the use of the lambert $w$ function in the stability analysis of time-delay systems. Automatica, 41(11), 1979-1985.

Hwang, C., \& Cheng, Y. C. (2006). A numerical algorithm for stability testing of fractional delay systems. Automatica, 42(5), 825-831.

Mäkilä, P. M., \& Partington, J. R. (1993). Robust stabilization-bibo stability, distance notions and robustness optimization. Automatica, 23(3), 681-693.

Marshall, J. E., Górecki, H., Korytowski, A., \& Walton, K. (1992). Time-delay systems: stability and performance criteria with applications. Ellis Horwood.

Matignon, D. (1998). Stability properties for generalized fractional differential systems. ESAIM: Proceedings, 5, 145-158.

Niculescu, S.-I. (2001). Delay effects on stability, a robust control approach. In LNCIS: Vol. 269. London: Springer-Verlag.

Niculescu, S.-I., \& Gu, K. (2004). Advances in time-delay systems. Springer.

Olgac, N., \& Sipahi, R. (2002). An exact method for the stability analysis of time delayed LTI systems. IEEE Transactions on Automatic Control, 47(5), 793-797.
Olgac, N., \& Sipahi, R. (2004). A practical method for analyzing the stability of neutral type LTI-time delayed systems. Automatica, 40, 847-853.

Ozturk, N., \& Uraz, A. (1985). An analysis stability test for a certain class of distributed parameter systems with delays. IEEE Transactions on Circuits and Systems, 32(4), 393-396.

Partington, J. R., \& Bonnet, C. (2004). $H_{\infty}$ and BIBO stabilization of delay systems of neutral type. Systems and Control Letters, 52, 283-288.

Partington, J. R., \& Mäkilä, P. M. (1994). Worst-case analysis of identification-bibo robustness for closed-loop data. IEEE Transactions on Automatic Control, 39, 2171-2176.

Pontryagin, L. S. (1955). On the zeros of some elementary transcendental functions. American Mathematical Society Translations, 2(1), 95-110.

Richard, J. P. (2003). Time-delay systems: an overview of some recent advances and open problems. Automatica, 39, 1667-1694.

Walton, K., \& Marshall, J. E. (1987). Direct method for tds stability analysis. IEE Proceedings D (Control Theory and Applications), 134, 101-107.

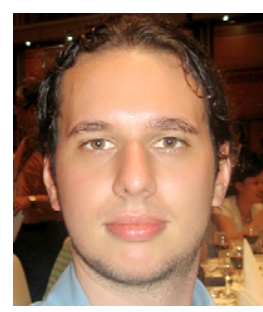

André Ricardo Fioravanti was born in São Paulo, Brazil in 1982. He received the B.S. and M.S. degrees in electrical engineering from the School of Electrical and Computer Engineering, University of Campinas (UNICAMP), Campinas, Brazil, in 2006 and 2007, respectively. He received the Ph.D. degree from the University of Paris XI, in 2011.

Since 2011, he has been a Research Associate with FEEC/UNICAMP. His research interests include convex programming, numerical methods, control and filtering of time-delay and Markovian systems.

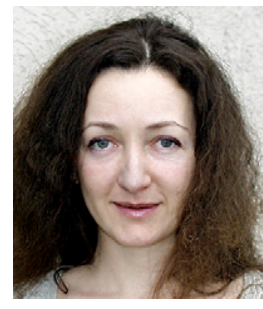

Catherine Bonnet was born in Marseille, France, in 1964. She obtained her M.S. degree in 1985 and her Ph.D. degree in 1991 both in Applied Mathematics at the University of Provence, Marseille and her French Habilitation (HDR) in Mathematics at the University of Paris 6 in 2008.

From 1986 to 1989 she worked as a research fellow with the Aerospatiale company. She was a temporary lecturer in Aix-Marseille Universities from 1990 to 1993 and then a postdoc at the University of Leeds, UK and a postdoc at Inria Rocquencourt, France where she became a researcher in 1994.

In 2010, she joined Inria Saclay-Île-de-France where she is currently leading the DISCO team.

Her research interests include approximation, robust control, delay and fractional systems as well as modeling in medicine.

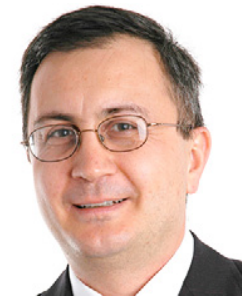

Hitay Özbay is a Professor of Electrical and Electronics Engineering at Bilkent University (Ankara, Turkey). He received the B.Sc. degree in Electrical Engineering from Middle East Technical University (Ankara, Turkey) in 1985, the M.Eng degree in Electrical Engineering from McGill University (Montreal, Canada) in 1987, and the Ph.D. degree in Control Sciences and Dynamical Systems from the University of Minnesota (Minneapolis, USA) in 1989. Dr. Özbay was with the University of Rhode Island (1989-1990) and The Ohio State University (1991-2006), where he was a Professor of Electrical and Computer Engineering, prior to joining Bilkent University in 2002, on leave from OSU. He served as an Associate Editor on the Editorial Board of the IEEE Transactions on Automatic Control (1997-1999), and Automatica, (2001-2007). He was a member of the Board of Governors of the IEEE Control Systems Society (1999) and a vicechair of the IFAC Technical Committee on Networked Control Systems (2005-2011).

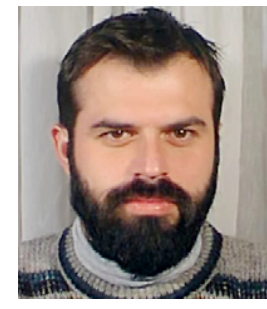

Silviu-Iulian Niculescu received the B.S. degree from the Polytechnical Institute of Bucharest, Romania, the M.Sc. and Ph.D. degrees from the Institut National Polytechnique de Grenoble, France, and the French Habilitation (HDR) from Université de Technologie de Compiègne, all in Automatic Control, in 1992, 1993, 1996, and 2003, respectively. He is currently Research Director at the CNRS (French National Center for Scientific Research) and the head of L2S (Laboratory of Signals and Systems). He is also the responsible of the IFAC Research Group on "Timedelay systems" since its creation in October 2007. He is a member of IFAC Technical Committee on Linear Systems (since 2002) and of the IPC of 33 International Conferences. He served as an Associate Editor of the IEEE Transactions on Automatic Control (2003-2005). Since 2011, he is an Associate Editor of European Journal of Control and IMA Journal of Mathematical Control and Information. He is author/co-author of 3 books and more than 325 scientific peer reviewed papers; his research interests include delay systems, robust control, operator theory, and numerical methods in optimization, and their applications to the design of engineering systems. He was awarded the French CNRS bronze and silver medals for the scientific research in 2001 and 2011, respectively. For further information, see http://silviu.niculescu.lss.supelec.fr. 